\title{
The Circulatory System Of Decapod Crustaceans And Its Functional Role In Cardiovascular Dynamics.
}

\section{J. McGaw}

Department of Biological Sciences, University of Nevada, Las Vegas, 4505 Maryland Parkway, Las Vegas, NV 89154-4004.

The decapod crustacean circulatory system has received extensive investigation during the last decade 1,2 . Despite our increased understanding of physiological control mechanisms, recent work on the gross anatomy of this system is still scant. A number of classical papers describe the anatomy of the decapod crustacean system in detail 3,4 . However, most of these articles were written at the beginning of the last century or the end of the previous century before the advent of modern imaging techniques allowed the detailed analysis of the circulatory system to be resolved.

In the present study the circulatory system of adult decapod crustaceans, was mapped using corrosion cast techniques. Batsons Monomer (Polysciences Inc) was thinned with methyl acrylate methyl ester. It was injected into the pericardial sinus of live crustaceans using a syringe pump. A small cut was made in the arthrodial membrane of a pereiopod to allow hemolymph to escape during resin perfusion. Following injection, the animals were flash frozen in liquid nitrogen. The soft tissue was then macerated, over a 3 day period, in a saturated solution of potassium hydroxide. The remaining resin cast and chitinous exoskeleton, was washed and then dried at room temperature. The chitinous tissue was removed by soaking in $1 \mathrm{M}$ solution of hydrochloric acid for 6 hours. This left a corrosion cast of the circulatory system without the need for any manual removal of tissue, which could damage the fine vessels. In order to check the validity of this method, the circulatory system was also mapped by injecting a suspension of barium sulfate into the pericardial sinus, followed by radiography of the specimens.

Seven arteries (five arterial systems) arise from the heart. The anterior aorta exits from the anterior dorsal surface of the heart and branches into 3 arteries. The paired optic arteries arise at right angles and supply hemolymph to the eyestalks. The small cerebral artery, which branches laterally from the main artery, supplies hemolymph to the supraesophageal ganglion. Hemolymph flow into this structure may be aided, in part, by a small accessory pump, the cor frontale. The paired anterolateral arteries also exit from the anterior dorsal surface of the heart; these arteries and their sub-branches supply hemolymph to the gonads, hepatopancreas, stomach, antennal gland, mandibular muscles and the hypodermis of the anterior cephalothorax. The paired hepatic arteries exit the heart anteriorly and ventrally and branch profusely within the hepatopancreas. A smaller side branch, the pyloric hepatic artery supplies hemolymph to the pyloric stomach and midgut. The smallest artery, the posterior aorta, branches off the posterior ventral surface of the heart; it joins with the inferior abdominal artery in the region of the second abdominal segment 
and these arteries supply hemolymph to the hindgut and abdomen. The largest artery is the sternal artery, which exits from the ventral surface of the heart; the ventral thoracic artery branches off the sternal artery and supplies hemolymph to the chelae, the mouthparts and to each pereiopod.

The present study shows that the circulatory system is highly developed, with arteries dividing into smaller capillary-like vessels that ramify profusely within individual organs. The return vessels, the sinuses, are discrete channels rather than random open spaces as previously described. The presence of these sinuses, rather than a complete venous return system, defines the system as an open circulatory system. However, the terms open and closed are ambiguous. The systems of cladoceran, anostracan, calanoid, and cirriped crustaceans are definitely classed as open, because they lack the discrete vessels of the decapod crustaceans 5 . The decapod crustacean system, however, is far more complex, with hydrostatic pressures that rival those of some lower vertebrate systems 1 and neurohormonal control mechanisms that allow fine regulation of cardiac function and regional hemolymph flow 6 . This study suggests that there may be room for a third category of circulatory system, one that would define the decapod crustacean circulatory system as Apartially closed@ rather than open. Thus the present study refines and advances descriptions of the circulatory system and is discussed in relation to recent work on hemolymph flow in crustaceans.

This study also invites further investigation into whether the decapod crustacean circulatory system has a two fluid-compartment system (intracellular and hemolymph), or a more vertebrate-like three-fluid-compartment system (intracellular, interstitial, and blood). Because the vascular system of decapods in general, is recognized for its complexities, further examinations of the local or central regulatory capabilities of regional blood flow should also support the regulatory comparisons made between decapods and lower vertebrates 7 .

\section{References}

1 . L.E. Burnett \& B.R. McMahon. Physiol. Zool. 63 (1990) 35.

2 . I.J. McGaw \& C.L. Reiber. J. Morphol. 251. (2002) 1.

3 . C. Claus. Ar Zool. Inst Univ. Wien U Zool. Sta. Triest. 5 (1884) 271.

4 . J. Pearson. Livpl. Mar. Biol. Mem. 16 (1908) 1.

5 . P.A. McLaughlin. Biology of Crustacea. Vol 5 (1983) 1.

6 . I.J. McGaw \& B.R. McMahon. J. Crust. Biol. 19 (1999) 435.

7 . This work was supported by the Office of Research at UNLV. The aid of Dr Carl Reiber, UNLV Biological Sciences, is gratefully acknowledged 